\title{
Perturbation perspective of partial coherence discussion on imperfect $x-$ ray optical elements
}

Lingfei Hu, John Sutter, Hongchang Wang, Kawal Sawhney

Lingfei Hu, John P. Sutter, Hongchang Wang, Kawal Sawhney, "Perturbation perspective of partial coherence discussion on imperfect x-ray optical elements," Proc. SPIE 11493, Advances in Computational Methods for X-Ray Optics V, 114930D (21 August 2020); doi: 10.1117/12.2567207

SPIE. Event: SPIE Optical Engineering + Applications, 2020, Online Only 


\title{
Perturbation perspective of partial coherence discussion on imperfect $\mathrm{X}$-ray optical elements
}

\author{
Lingfei Hu*a, John P Sutter ${ }^{\mathrm{a}}$, Hongchang Wang ${ }^{\mathrm{a}}$, Kawal Sawhney ${ }^{\mathrm{a}}$ \\ ${ }^{a}$ Diamond Light Source Ltd., Harwell Science and Innovation Campus, Didcot, OX11 0DE, UK
}

\begin{abstract}
The rapid development of new-generation synchrotron facilities with excellent coherence demands more accurate evaluation of beamline performance. A perturbation theory based on wave optics is proposed in this work to describe the effect of imperfections on the performance of X-ray optical elements. It shows that the perturbed performance of the non-ideal optical element could be derived from the perfect performance of the ideal optic through a convolution operation. The semi-analytical approach proposed here provides a new way to improve the simulation efficiency for imperfect optical elements. The finite aperture effect on diffraction-limited optics and focal shape distortion by surface height error are treated to show the application of the proposed method.
\end{abstract}

Keywords: X-ray optics, wave optics, partial coherence, wavefront propagation, beamline design

\section{INTRODUCTION}

As more and more low emittance sources are been built or planned ${ }^{1-5}$, the coherence of the X-ray beam starts to play an important role. However, the great advances of coherent X-ray sources make the tolerances on optical elements more stringent if the high coherence originating at the source is to be preserved throughout the beamline. It is widely accepted that for highly coherent sources, such as diffraction-limited synchrotron radiation (DLSR) or X-ray free electron lasers (FEL), the wave optics needs to be taken into consideration. Wave optics simulation codes calculate wavefront propagation using the Fresnel diffraction integral. Several types of methods for wavefront propagation calculation have been used by different simulation codes. Among them are the Fourier-optics-based code SRW ${ }^{6}$, stationary-phaseapproximation-based code PHASE ${ }^{7}$, hybrid-method-based codes OASYS ${ }^{8,9}$ and $\mathrm{xrt}^{10}$, etc. Although wave optics simulations yield more accurate results than ray tracing for DLSR and X-ray FELs, they also require substantially more computational resources. Some improvements ${ }^{11,12}$ have been done to make wave optics simulations less computationally demanding.

In this work, a theoretical approach to evaluate the optical performance distortion caused by imperfect optical elements is given. The proposed theory could be used to evaluate the impacts of finite size aperture, surface height error and other imperfections of optical elements as long as they can be described by a complex transfer function. The proposed theory provides a perspective to regard the imperfection's influence as a "perturbation" to convolve with its ideal optical performance. The proposed theoretical framework is universal. It can deal with various SR source models such as Gaussian-Schell model or more rigorous undulator models. In addition, the existing measures ${ }^{11,12}$ to reduce the computation load can be integrated into this framework. It is shown in this paper that the coherent mode decomposition can be used to deal with the 4D convolution which comes from the proposed theory. As a result, this theoretical framework could be potentially applied to new simulation software designs to improve current computation efficiency in the future.

In section 2 we give the detailed derivation of the proposed theoretical framework. Two examples of the performance distortion of a mirror, one from its finite aperture and one from its surface height error, are given in section 3 to show the application of the proposed theory.

*lingfei.hu@diamond.ac.uk; phone +44 (0)1235 394075; 


\section{PERTURBATION THEORY FOR PARTIALLY COHERENT BEAM}

\subsection{Basic concepts}

The coherence properties of X-rays can be well described by the mutual coherence function and other related functions derived from it ${ }^{13}$. The mutual coherence function characterizes the time-space correlation of the wavefield at two timespace coordinates $\left(\mathbf{r}_{1}, t\right)$ and $\left(\mathbf{r}_{2}, t+\tau\right)$. The mutual coherence function $\left(\mathbf{r}_{1}, \mathbf{r}_{2} ; t+\tau\right)$ is defined as:

$$
\Gamma\left(\mathbf{r}_{1}, \mathbf{r}_{2} ; \tau\right)=\left\langle E\left(\mathbf{r}_{1}, t+\tau\right) E^{*}\left(\mathbf{r}_{2}, t\right)\right\rangle_{T}
$$

where the bracket $\langle\ldots\rangle_{T}$ means time averaging. $E(\mathbf{r}, t)$ is the complex amplitude of the wavefield. We discuss only the spatial (transverse) coherence in this work. It is convenient to introduce the cross spectral density (CSD), which can be defined as the Fourier transform of the mutual coherence function:

$$
G\left(\mathbf{r}_{1}, \mathbf{r}_{2} ; \omega\right)=\int_{-\infty}^{\infty} \Gamma\left(\mathbf{r}_{1}, \mathbf{r}_{2} ; t\right) \exp (i \omega t) d t
$$

where $\omega$ is the frequency of the radiation. By definition, when the two spatial coordinates $\mathbf{r}_{1}$ and $\mathbf{r}_{2}$ are set to be equal, CSD function represents the intensity at the spatial position, $G\left(\mathbf{r}_{1}, \mathbf{r}_{1} ; \omega\right)=I\left(\mathbf{r}_{1}\right)$. The normalized CSD function is also important and defined as spectral degree of coherence (SDC):

$$
\mu\left(\mathbf{r}_{1}, \mathbf{r}_{2} ; \omega\right)=\frac{G\left(\mathbf{r}_{1}, \mathbf{r}_{2} ; \omega\right)}{\sqrt{G\left(\mathbf{r}_{1}, \mathbf{r}_{1} ; \omega\right)} \sqrt{G\left(\mathbf{r}_{2}, \mathbf{r}_{2} ; \omega\right)}}=\frac{G\left(\mathbf{r}_{1}, \mathbf{r}_{2} ; \omega\right)}{\sqrt{I\left(\mathbf{r}_{1} ; \omega\right)} \sqrt{I\left(\mathbf{r}_{2} ; \omega\right)}}
$$

Hereafter, we will discuss the spectral functions, omitting the $\omega$ for simplicity.

It is widely known that the free space propagation of the CSD function obeys the following relation ${ }^{14}$ :

$$
G_{z}\left(\mathbf{r}_{1}, \mathbf{r}_{2} ; z=z_{0}\right)=\int G_{0}\left(\mathbf{r}_{1}^{\prime}, \mathbf{r}_{2}^{\prime} ; z=0\right) K_{z}\left(\mathbf{r}_{1}-\mathbf{r}_{1}^{\prime}\right) K_{z}^{*}\left(\mathbf{r}_{2}-\mathbf{r}_{2}^{\prime}\right) d^{2} \mathbf{r}_{1}^{\prime} d^{2} \mathbf{r}_{2}^{\prime}
$$

where $K_{z}(\mathbf{r})$ is the Fresnel propagator along the optical axis, $z=0$ is the initial plane, $z=z_{0}$ is the final observing plane. For synchrotron radiation or FEL beamlines, the paraxial approximation is always satisfied. Thus, the Fresnel propagator $K_{\mathrm{z}}(\mathbf{r})$ has the following expression:

$$
K_{z}(\mathbf{r})=-\frac{i}{\lambda z} \exp \left(i k \frac{\mathbf{r}^{2}}{2 z}\right)
$$

Under the assumption of paraxial approximation, the free space propagation of CSD function has the following form:

$$
G_{0 z}\left(\mathbf{r}_{1}, \mathbf{r}_{2}\right)=\frac{1}{\lambda^{2} z^{2}} \iint G_{0}\left(\mathbf{r}_{1}^{\prime}, \mathbf{r}_{2}^{\prime}\right) \exp \left[i k \frac{\left(\mathbf{r}_{1}-\mathbf{r}_{1}^{\prime}\right)^{2}}{2 z}\right] \exp \left[-i k \frac{\left(\mathbf{r}_{2}-\mathbf{r}_{2}^{\prime}\right)^{2}}{2 z}\right] d \mathbf{r}_{1}^{\prime} d \mathbf{r}_{2}^{\prime}
$$

In the above equation, the spatial coordinate $\mathbf{r}_{1}^{\prime}, \mathbf{r}_{2}^{\prime}$ represent the positions in the initial plane immediately after the ideal optical elements or at the secondary source plane. The spatial coordinate $\mathbf{r}_{1}, \mathbf{r}_{2}$ are in the observing plane, which is usually considered to be the sample plane or the focal plane of the final optical elements. No optical elements are present between the initial plane and focal plane. In addition, $G_{0}\left(\mathbf{r}_{1}, \mathbf{r}_{2}\right)$ is the CSD function right after the ideal optical surface, $z$ is the distance between the initial plane and the observing plane, $\lambda$ is the wavelength of the beam, $k=2 \pi / \lambda$ is the wave vector. As a result, $G_{0 z}\left(\mathbf{r}_{1}, \mathbf{r}_{2}\right)$ represents the ideal CSD function in the observing plane. The ideal CSD function means that it goes through perfect optical elements. An ideal optical element may be defined as a surface of infinite extent with the ideal physical shape for beam profile shaping. For example, an ideal infinite plane mirror only deflects the incident beam such that the reflected beam propagates as a free space diffraction along its reflected direction. An ideal infinite focusing mirror images the source according to its demagnification factor.

Every real optical element is imperfect. The impact from various types of imperfection can be expressed as a different complex transfer function $t\left(\mathbf{r}^{\prime}\right)$. The complex transfer function $t\left(\mathbf{r}^{\prime}\right)$ can represent partial transmission due to mirror 
reflectivity, finite mirror size, mirror surface height error, etc. When the imperfection of the optical element is taken into consideration, the resulting distorted CSD function obeys a relation similar to Equation (6):

$$
G_{z}\left(\mathbf{r}_{1}, \mathbf{r}_{2}\right)=\frac{1}{\lambda^{2} z^{2}} \iint G_{0}\left(\mathbf{r}_{1}^{\prime}, \mathbf{r}_{2}^{\prime}\right) t\left(\mathbf{r}_{1}^{\prime}\right) t^{*}\left(\mathbf{r}_{2}^{\prime}\right) \exp \left[i k \frac{\left(\mathbf{r}_{1}-\mathbf{r}_{1}^{\prime}\right)^{2}}{2 z}\right] \exp \left[-i k \frac{\left(\mathbf{r}_{2}-\mathbf{r}_{2}^{\prime}\right)^{2}}{2 z}\right] d \mathbf{r}_{1}^{\prime} d \mathbf{r}_{2}^{\prime}
$$

\subsection{Perturbation perspective of the imperfect CSD function propagation}

The basic idea of our new treatment is to compare the distorted CSD function, $G_{z}\left(\mathbf{r}_{1}, \mathbf{r}_{2}\right)$, from equation (7) to the ideal one, $G_{0 z}\left(\mathbf{r}_{1}, \mathbf{r}_{2}\right)$, from equation (6) in order to set the relation between these two CSD functions. Notice that equation (6) could be written as

$$
\begin{aligned}
& G_{0 z}\left(\mathbf{r}_{1}, \mathbf{r}_{2}\right) \\
& =\frac{1}{\lambda^{2} z^{2}} \exp \left(i k \frac{\mathbf{r}_{1}^{2}-\mathbf{r}_{2}^{2}}{2 z}\right) F_{\left.\frac{\mathbf{r}_{2}}{\lambda z}\right|_{\mathbf{r}_{2}^{\prime}} ^{-1}}\left[F_{\left.\frac{\mathbf{r}_{1}}{\lambda z}\right|_{\mathbf{r}_{1}^{\prime}}}\left[G_{0}\left(\mathbf{r}_{1}^{\prime}, \mathbf{r}_{2}^{\prime}\right) \exp \left(i k \frac{\mathbf{r}_{1}^{\prime 2}}{2 z}\right) \exp \left(-i k \frac{\mathbf{r}_{2}^{\prime 2}}{2 z}\right)\right]\right]
\end{aligned}
$$

In the above equation, $\left.F_{\frac{\mathbf{r}_{1}}{\lambda z}}\right|_{\mathbf{r}_{1}^{\prime}}$ and $\left.F_{\frac{\mathbf{r}_{2}}{\lambda z}}^{-1}\right|_{\mathbf{r}_{2}^{\prime}}$ are 2D Fourier and inverse Fourier transform, respectively. $F_{\frac{\mathbf{r}_{1}}{\lambda z}}$, means performing the Fourier transform from $\left.\mathbf{r}_{1}^{\prime}=\left(x_{1}^{\prime}, y_{1}\right)^{\prime}\right)$ into spatial frequency $\left(x_{1} / \lambda z, y_{1} / \lambda z\right) .\left.F_{\frac{\mathbf{r}_{2}}{\lambda z}}^{-1}\right|_{\mathbf{r}_{2}^{\prime}} ^{\prime}{ }^{\prime}$ means performing the Fourier transform from $\mathbf{r}_{2}^{\prime}=\left(x_{2}^{\prime}, y_{2}^{\prime}\right)$ into spatial frequency $\left(x_{2} / \lambda z, y_{2} / \lambda z\right)$. The $2 \mathrm{D}$ Fourier transform and inverse Fourier transform are defined as

$$
\begin{aligned}
& F_{\frac{\mathbf{r}_{1}}{\lambda z}}\left[f\left(\mathbf{r}_{1}^{\prime}\right)\right]=\int_{-\infty}^{\infty} \int_{\mathbf{r}_{1}^{\prime}}^{\infty} f\left(x_{1}^{\prime}, y_{1}^{\prime}\right) \exp \left[-i 2 \pi\left(x_{1}^{\prime} \frac{x_{1}}{\lambda z}+y_{1}^{\prime} \frac{y_{1}}{\lambda z}\right)\right] d x_{1}^{\prime} d y_{1}^{\prime} \\
& F_{\frac{\mathbf{r}_{2}}{\lambda z}}^{-1}\left[f\left(\mathbf{r}_{2}^{\prime}\right)\right]=\int_{-\infty}^{\infty} \int_{-\infty}^{\infty} f\left(x_{2}^{\prime}, y_{2}^{\prime}\right) \exp \left[i 2 \pi\left(x_{2}^{\prime} \frac{x_{2}}{\lambda z}+y_{2}^{\prime} \frac{y_{2}}{\lambda z}\right)\right] d x_{2}^{\prime} d y_{2}^{\prime}
\end{aligned}
$$

Similarly, equation (7) has the following form

$$
\begin{aligned}
& G_{0 z}\left(\mathbf{r}_{1}, \mathbf{r}_{2}\right) \\
& =\frac{1}{\lambda^{2} z^{2}} \exp \left(i k \frac{\mathbf{r}_{1}^{2}-\mathbf{r}_{2}^{2}}{2 z}\right) F_{\left.\frac{\mathbf{r}_{2}}{\lambda z}\right|_{\mathbf{r}_{2}^{\prime}} ^{-1}}\left[F_{\frac{\mathbf{r}_{1}}{\lambda z} \mid r_{\mathbf{r}_{1}^{\prime}}}\left[G_{0}\left(\mathbf{r}_{1}^{\prime}, \mathbf{r}_{2}^{\prime}\right) t\left(\mathbf{r}_{1}^{\prime}\right) t^{*}\left(\mathbf{r}_{2}^{\prime}\right) \exp \left(i k \frac{\mathbf{r}_{1}^{\prime 2}}{2 z}\right) \exp \left(-i k \frac{\mathbf{r}_{2}^{\prime 2}}{2 z}\right)\right]\right]
\end{aligned}
$$

Applying the convolution theorem ${ }^{15}$ repeatedly and interchanging the integration order of equation (10), we have: 


$$
\begin{aligned}
& G_{z}\left(\mathbf{r}_{1}, \mathbf{r}_{2}\right) \\
& =\frac{1}{\lambda^{2} z^{2}} \exp \left(i k \frac{\mathbf{r}_{1}^{2}-\mathbf{r}_{2}^{2}}{2 z}\right) \cdot\left\{F _ { \frac { \mathbf { r } _ { 2 } } { \lambda z } | _ { \mathbf { r } _ { 2 } ^ { \prime } } ^ { - 1 } } \left[F_{\left.\frac{\mathbf{r}_{1}}{\lambda z}\right|_{\mathbf{r}_{1}^{\prime}}}\left[G_{0}\left(\mathbf{r}_{1}^{\prime}, \mathbf{r}_{2}^{\prime}\right) \exp \left(i k \frac{\mathbf{r}_{1}^{\prime 2}}{2 z}\right) \exp \left(-i k \frac{\mathbf{r}_{2}^{\prime 2}}{2 z}\right)\right] \otimes\right.\right. \\
& F_{\left.\frac{\mathbf{r}_{2}}{\lambda z}\right|_{\mathbf{r}_{2}^{\prime}} ^{-1}}\left[F_{\left.\frac{\mathbf{r}_{1}}{\lambda z}\right|_{\mathbf{r}_{1}^{\prime}}}\left[t\left(\mathbf{r}_{1}^{\prime}\right) t^{*}\left(\mathbf{r}_{2}^{\prime}\right)\right]\right\}
\end{aligned}
$$

The “ $\otimes ”$ denotes 4D convolution:

$$
f\left(\mathbf{r}_{1}^{\prime}, \mathbf{r}_{2}^{\prime}\right) \otimes g\left(\mathbf{r}_{1}^{\prime}, \mathbf{r}_{2}^{\prime}\right)=\iiint \int f\left(\xi_{1}, \eta_{1}, \xi_{2}, \eta_{2}\right) g\left(x_{1}^{\prime}-\xi_{1}, y_{1}^{\prime}-\eta_{1}, x_{2}^{\prime}-\xi_{2}, y_{2}^{\prime}-\eta_{2}\right) d \xi_{1} d \eta_{1} d \xi_{2} d \eta_{2}
$$

Comparing equation (8) and equation (11), the ideal CSD function at the focal plane and the distorted CSD function have the following relation:

$$
G_{z}\left(\mathbf{r}_{1}, \mathbf{r}_{2}\right) \exp \left(-i k \frac{\mathbf{r}_{1}^{2}-\mathbf{r}_{2}^{2}}{2 z}\right)=\left[G_{0 z}\left(\mathbf{r}_{1}, \mathbf{r}_{2}\right) \exp \left(-i k \frac{\mathbf{r}_{1}^{2}-\mathbf{r}_{2}^{2}}{2 z}\right)\right] \otimes F_{\left.\frac{\mathbf{r}_{2}}{\lambda z}\right|_{\mathbf{r}_{2}^{\prime}} ^{-1}}\left[F_{\left.\frac{\mathbf{r}_{1}}{\lambda z}\right|_{\mathbf{r}_{1}^{\prime}}}\left[t\left(\mathbf{r}_{1}^{\prime}\right) t^{*}\left(\mathbf{r}_{2}^{\prime}\right)\right]\right]
$$

Equation (13) provides us a perturbation perspective to view the optical performance degradation due to the imperfections of the optics. According to equation (13), the impact from the imperfect optical elements can be treated as a perturbation to the ideal optical performance. This perturbation interacts with the ideal performance through a convolution operation.

In order to use equation (13) to explore the impact that comes from optical imperfections, we need the ideal performance of the perfect optical element. A perfect optical system images the source according to the magnification factor $M$. No loss of intensity and no distortion of the intensity profile will occur at the focal plane if all the elements are perfect. As long as the source information is known, the CSD function at the observing plane can be deduced from the source according to the demagnification factor of the optical layout.

\subsection{Reducing computational consumption by means of coherent mode decomposition}

Equation (13) uses a 4D convolution for calculation. In the worst case, if all the coordinates are coupled with each other, 4D integration is unavoidable. This 4D integration requires vast computer memory and therefore suffers from very slow computation speed if limited memory is available. We propose to use coherent mode decomposition ${ }^{12,13}$ to reduce the memory required for the convolution in equation (13).

The CSD function $G_{0 z}\left(\mathbf{r}_{1}, \mathbf{r}_{2}\right)$ can be decomposed into a sum over independent coherent modes ${ }^{13}$ :

$$
G_{0 z}\left(\mathbf{r}_{1}, \mathbf{r}_{2}\right)=\sum_{m=0}^{+\infty} \lambda_{m} \Phi_{m}^{*}\left(\mathbf{r}_{1}\right) \Phi_{m}\left(\mathbf{r}_{2}\right)
$$

where $\lambda_{m}$ are the eigenvalues and $\Phi_{m}$ are the eigenfunctions or coherent modes. Using coherent mode decomposition, the two spatial coordinates in equation (13) are decoupled: 


$$
\begin{aligned}
& G_{z}\left(\mathbf{r}_{1}, \mathbf{r}_{2}\right) \exp \left(-i k \frac{\mathbf{r}_{1}^{2}-\mathbf{r}_{2}^{2}}{2 z}\right) \\
& \left.=\sum_{m=0}^{+\infty} \lambda_{m}\left\{\left[\Phi_{m}^{*}\left(\mathbf{r}_{1}\right) \exp \left(-i k \frac{\mathbf{r}_{1}^{2}}{2 z}\right)\right] * F_{\left.\frac{\mathbf{r}_{1}}{\lambda z}\right|_{\mathbf{r}_{1}^{\prime}}}\left[t\left(\mathbf{r}_{1}^{\prime}\right)\right]\right\}\left\{\Phi_{m}\left(\mathbf{r}_{2}\right) \exp \left(i k \frac{\mathbf{r}_{2}^{2}}{2 z}\right)\right] * F_{\frac{\mathbf{r}_{2}}{\lambda z} \mid}^{-1},\left[t^{*}\left(\mathbf{r}_{2}^{\prime}\right)\right]\right\}
\end{aligned}
$$

The “*” denotes 2D convolution:

$$
f\left(\mathbf{r}_{1}\right) * g\left(\mathbf{r}_{1}\right)=\iint f\left(\xi_{1}, \eta_{1}\right) g\left(x_{1}-\xi_{1}, y_{1}-\eta_{1}\right) d \xi_{1} d \eta_{1}
$$

The previous 4D integration reduces to the outer product of two 2D "images" according to equation (15). Another advantage of equation (15) is that the fast Fourier transform (FFT) can be used not only for the complex transfer functions, but also for the convolution operations through the convolution theorem ${ }^{15}$.

\section{SIMULATION}

\subsection{Source parameters and optical layout for simulation}

Figure 1 shows a typical optical layout for a nano-focusing beamline. The source is directly focused in the vertical direction using an elliptical mirror (VKB). The source to mirror distance is $207 \mathrm{~m}$ and the mirror to image distance is $0.36 \mathrm{~m}$. The demagnification factor $M_{y}$ in the vertical direction is 575. In the horizontal direction, the source goes through two focusing mirrors before it hits the sample. A horizontally focusing mirror (HFM) images the source onto a secondary source slit. An elliptical mirror (HKB) then produces an image of the secondary source. The secondary source has the same size as the undulator source. The distance from the secondary source to the horizontal focusing mirror HKB is $130.25 \mathrm{~m}$, and the mirror to image distance is $0.11 \mathrm{~m}$. The demagnification factor $M_{x}$ in the horizontal direction is 1184 . We will discuss the KB mirrors (HKB and VKB) in this work. The source used is from the High Energy Photon Source (HEPS) project ${ }^{3}$, see Table 1 for the parameters.

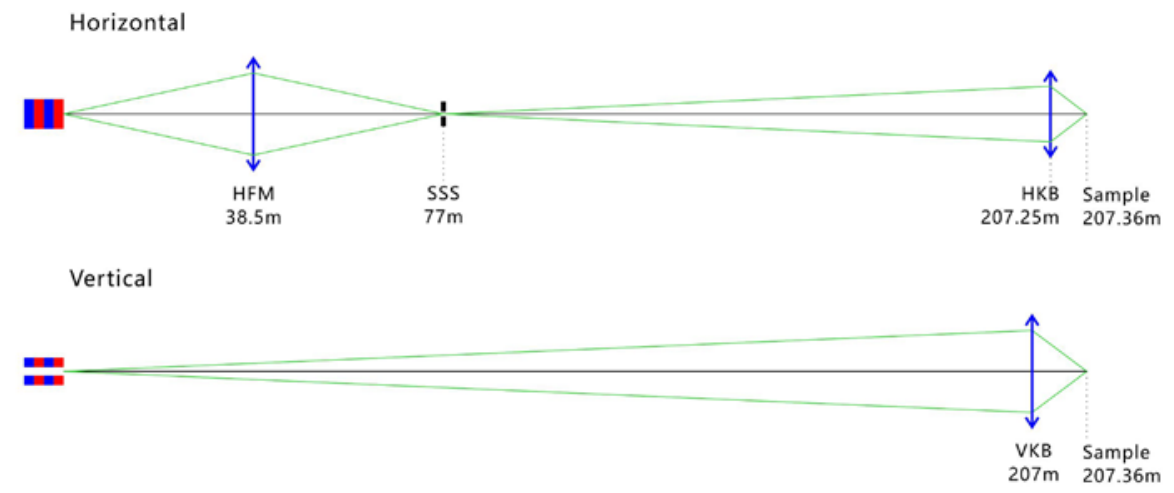

Figure 1. Typical layout of nano-focusing beamline. In the horizontal direction, the source is first imaged at the secondary source slit. The secondary source has the same horizontal size as the undulator source. The secondary source then is focused to the sample position by a second horizontal focusing mirror. In the vertical direction, the undulator source is directly focused to the sample plane.

Table 1. Source parameters.

\begin{tabular}{llll}
\hline Photon energy & $10 \mathrm{keV}$ & Source divergence $(\mathbf{H} \times \mathbf{V})$ & $4.97 \mu \mathrm{rad} \times 4.04 \mu \mathrm{rad}$ \\
Source size $(\mathbf{H} \times \mathbf{V})$ & $9.14 \mu \mathrm{m} \times 3.43 \mu \mathrm{m}$ & Coherence length $(\mathbf{H} \times \mathbf{V})$ & $4.07 \mu \mathrm{m} \times 6.96 \mu \mathrm{m}$ \\
\hline
\end{tabular}




\subsection{Gaussian Schell-model}

For the convenience of the discussion in this paper, we use the Gaussian Schell-Model (GSM) to describe the source ${ }^{16,17}$. The advantage of the GSM is that the CSD function of the source can be written analytically given just a few parameters describing the properties of the source.

The CSD function of a GSM source is:

$$
G_{0}\left(\mathbf{r}_{s 1}, \mathbf{r}_{s 2}\right)=I_{0}\left[\exp \left(-\frac{x_{s 1}^{2}+x_{s 2}^{2}}{4 \sigma_{s x}^{2}}-\frac{y_{s 1}^{2}+y_{s 2}^{2}}{4 \sigma_{s y}^{2}}\right)\right] \exp \left[-\frac{\left(x_{s 1}-x_{s 2}\right)^{2}}{2 \xi_{s x}^{2}}-\frac{\left(y_{s 1}-y_{s 2}\right)^{2}}{2 \xi_{s y}^{2}}\right]
$$

where $I_{0}$ represents the maximum intensity at the source, and $\sigma_{s x, s y}$ and $\xi_{s x, s y}$ represent the source's RMS size and coherence length in two directions, respectively. $x_{s 1}, x_{s 2}, y_{s 1}, y_{s 2}$ are the coordinates at the source plane. Within the framework of the GSM, these parameters have the following relation:

$$
\xi_{s x, s y}=\frac{2 \sigma_{s x, s y}}{\sqrt{4 k^{2} \sigma_{s x, s y}^{2} \sigma_{s x, s y}^{\prime 2}-1}}
$$

where $\sigma_{s x, s y}$ is the angular divergence of the source in two directions. Usually, the product $\sigma_{s x} \sigma_{s x}$ and $\sigma_{s y} \sigma_{s y}$ are defined as beam emittance. As discussed in previous, the CSD function of the wave transmitted by a perfect focusing element is affected only by the demagnification factors. The CSD function at the image plane of the perfect optical element may be written as:

$$
G_{0}\left(\mathbf{r}_{1}, \mathbf{r}_{2}\right)=I_{0}\left[\exp \left(-\frac{x_{1}^{2}+x_{2}^{2}}{4 \sigma_{x}^{2}}-\frac{y_{1}^{2}+y_{2}^{2}}{4 \sigma_{y}^{2}}\right)\right] \exp \left[-\frac{\left(x_{1}-x_{2}\right)^{2}}{2 \xi_{x}^{2}}-\frac{\left(y_{1}-y_{2}\right)^{2}}{2 \xi_{y}^{2}}\right]
$$

with $\sigma_{x, y}=M_{x, y} \sigma_{s x, s y}, \xi_{x, y}=M_{x, y} \xi_{s x, s y}, M_{x}$ and $M_{y}$ are the demagnification factors in two directions, $\mathbf{r}_{1}, \mathbf{r}_{2}$ are the coordinates at the observing plane. Figure 2 shows the perfect intensity distribution and two normalized CSD functions $G_{0}\left(x_{1}, x_{2}\right.$, $\left.y_{1}=y_{2}=0\right)$ and $G_{0}\left(x_{1}=x_{2}=0, y_{1}, y_{2}\right)$ along the central horizontal and vertical lines in the observing plane.
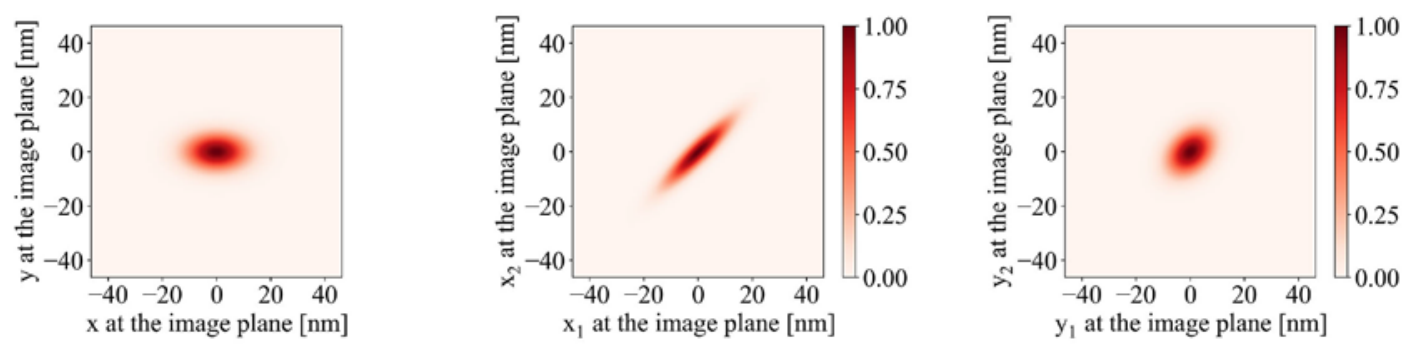

Figure 2. The ideal performance at the image plane. Left: The intensity distribution if all the optical elements are perfect. Middle: The normalized CSD function in horizontal central line. Right: The normalized CSD function in vertical central line.

\subsection{Finite aperture impact on diffraction limited optics}

Whereas a perfect optical element would have an infinitely large extent, a real optical surface always has a finite physical size. The complex transfer function $t\left(\mathbf{r}^{\prime}\right)$ to describe the finite optical element aperture at the exit plane can be expressed as a rectangular function:

$$
t\left(\mathbf{r}^{\prime}\right)=\operatorname{rect}\left(\frac{x^{\prime}}{L_{x}}\right) \operatorname{rect}\left(\frac{y^{\prime}}{L_{y}}\right)=\left\{\begin{array}{l}
1,-L_{x} / 2<x^{\prime}<L_{x} / 2 \text { and }-L_{y} / 2<y^{\prime}<L_{y} / 2 \\
0 . \text { elsewhere }
\end{array}\right.
$$


$L_{x, y}$ is the size of the optic aperture projected onto the plane immediately after the optical element. This plane is perpendicular to the propagation direction z. We examine the elliptical KB mirrors that form the second focusing system of Figure 1. The horizontal focusing mirror is $110 \mathrm{~mm}$ long with a grazing angle of $3.1 \mathrm{mrad}$ and the vertical focusing mirror is $360 \mathrm{~mm}$ long with the same grazing angle. Thus, $L_{x}$ is about $0.34 \mathrm{~mm}$ and $L_{y}$ is about $1.12 \mathrm{~mm}$. It is widely known that for the nano-focusing case, the final spot size is larger than the ideal value because of the broadening caused by the finite optical aperture. According to equation (13), the CSD function after the finite aperture $G_{z}\left(x_{1}, x_{2}\right)$ has the following form:

$$
\begin{aligned}
& G_{z}\left(\mathbf{r}_{1}, \mathbf{r}_{2}\right) \exp \left(-i k \frac{\mathbf{r}_{1}^{2}-\mathbf{r}_{2}^{2}}{2 z}\right) \\
& =\left[G_{0 z}\left(\mathbf{r}_{1}, \mathbf{r}_{2}\right) \exp \left(-i k \frac{\mathbf{r}_{1}^{2}-\mathbf{r}_{2}^{2}}{2 z}\right)\right] \otimes\left[L_{x}^{2} L_{y}^{2} \operatorname{sinc}\left(\frac{L_{x} x_{1}}{\lambda z}\right) \operatorname{sinc}\left(\frac{L_{x} x_{2}}{\lambda z}\right) \operatorname{sinc}\left(\frac{L_{y} y_{1}}{\lambda z}\right) \operatorname{sinc}\left(\frac{L_{y} y_{2}}{\lambda z}\right)\right]
\end{aligned}
$$

where $\operatorname{sinc}(x)=\sin (\pi x) / \pi x$. As discussed before, “ $\otimes$ ” denotes $4 \mathrm{D}$ convolution.

Figure3 shows the broadened intensity distribution and two CSD functions $G_{0}\left(x_{1}, x_{2}, y_{1}=y_{2}=0\right)$ and $G_{0}\left(x_{1}=x_{2}=0, y_{1}, y_{2}\right)$ in the observing plane. Both functions are broadened due to diffraction.
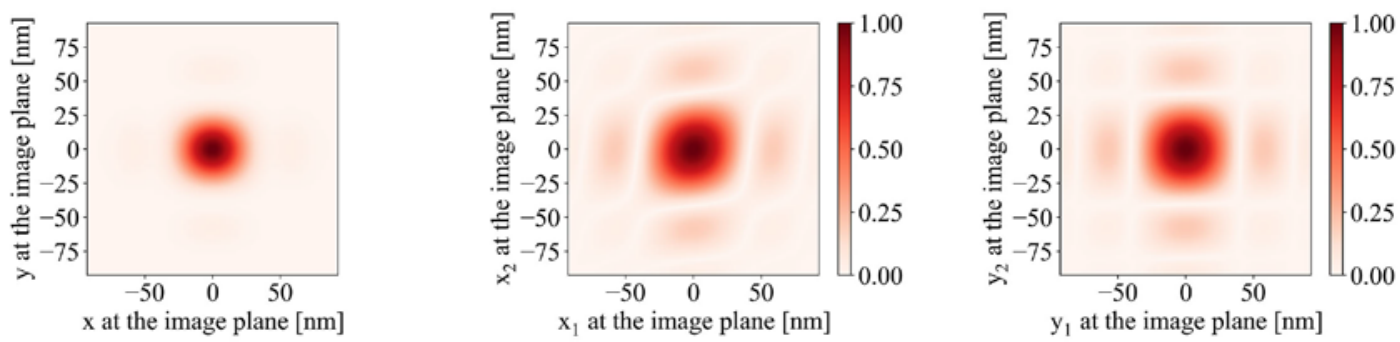

Figure 3. The distortion of the performance at the image plane caused by the finite aperture of the elliptical KB mirrors. Left: The broadened intensity distribution. Middle: The distorted CSD function along horizontal central cut line. Right: The distorted CSD function along vertical central cut line.

\subsection{Mirror surface height error impact}

It is well-known that the phase shift $\Delta \Phi_{h}$ due to the surface height error is:

$$
\Delta \Phi_{h}=-\frac{4 \pi}{\lambda} h\left(x_{s}\right) \sin \theta
$$

where $h\left(x_{s}\right)$ is the height error distribution along the mirror surface. To use equation (13), $x_{s}$ needs to be projected to the plane perpendicular to the optical axis by multiplying by the factor $\sin \theta$. We apply the height error to the horizontal focusing mirror HKB as shown in Figure 1. The surface height error impact on the horizontal perfect CSD function is: 


$$
\begin{aligned}
& G_{z}\left(\mathbf{r}_{1}, \mathbf{r}_{2}\right) \exp \left(-i k \frac{\mathbf{r}_{1}^{2}-\mathbf{r}_{2}^{2}}{2 z}\right) \\
& =\left[G_{0 z}\left(\mathbf{r}_{1}, \mathbf{r}_{2}\right) \exp \left(-i k \frac{\mathbf{r}_{1}^{2}-\mathbf{r}_{2}^{2}}{2 z}\right)\right] * \\
& F_{\left.\frac{x_{2}}{\lambda z}\right|_{x_{2}^{\prime}} ^{-1}}\left[F_{\left.\frac{x_{1}}{\lambda z}\right|_{x_{1}^{\prime}}}\left[\exp \left(-i \frac{4 \pi}{\lambda} h\left(x_{1}^{\prime}\right) \sin \theta\right) \exp \left(i \frac{4 \pi}{\lambda} h\left(x_{2}^{\prime}\right) \sin \theta\right)\right]\right]
\end{aligned}
$$

The meaning of symbol “*” is similar to the previous discussion but slightly different. The "*” in the above equation denotes 2D convolution with respect to the horizontal coordinates $x_{1}$ and $x_{2}$.

The left side of Figure 4 shows the height error distribution we used. The middle part of Figure 4 shows the 2D intensity distribution at the observing plane. The right side of Figure 4 shows the horizontal distorted intensity of the central cut at the observing plane. The perfect intensity is shown by the dashed curve. Compared with the ideal intensity, the distorted central intensity drops. The lost intensity forms some side peaks next to the main peak.
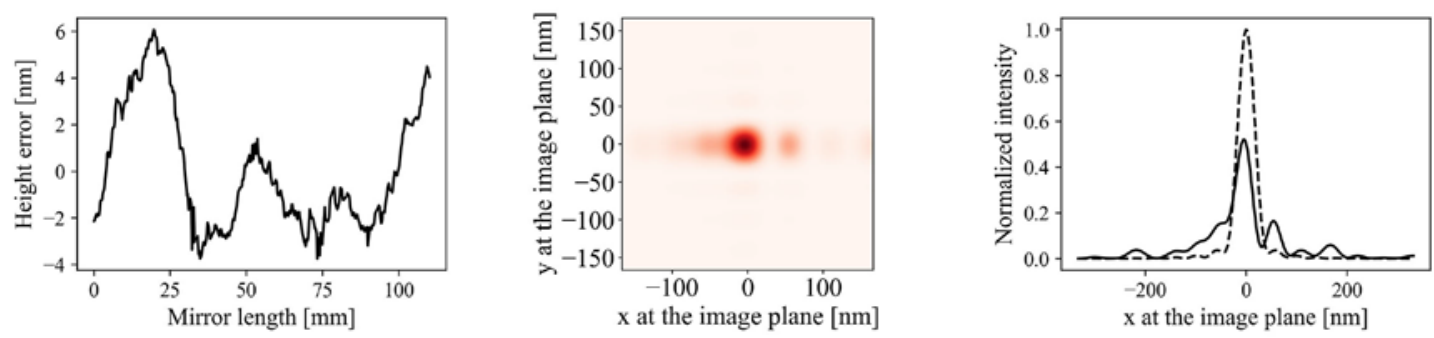

Figure 4. Left: The surface error distribution used for the calculation. Middle: The 2D intensity distribution due to the surface height error. Right: The distorted focal spot shape. The perfect focal spot is shown by the dashed curve.

\section{DISCUSSIONS AND CONCLUSIONS}

We have presented a universal theoretical framework for mutual coherence function calculations. This universal theoretical framework can deal with various synchrotron radiation source models such as the Gaussian Schell-model or more rigorous undulator models. This new theoretical framework provides us a new perspective to view the impacts of the imperfections from the optics. The proposed perspective regards the imperfection's impact as a "perturbation" that convolves with the optical element's ideal performance. To avoid the heavy computational load of the 4D integration that comes from the convolution, we have applied coherent mode decomposition. Two typical cases have been considered using the Gaussian Schell-model to show the application of the proposed theory. The finite aperture's impact on the diffraction-limited optics and the surface height error's influence on the highly coherent beam are common problems for beamline optics design. We have shown that the proposed theoretical framework can treat these problems successfully.

By separating the ideal CSD function from the impact of the imperfections, the time-consuming wave optics simulation can be performed more efficiently. The imperfection impacts can be easily evaluated using 2D FFT while the ideal CSD function only needs to be calculated once. By integrating the proposed theoretical framework into existing software, one could calculate the CSD functions distorted by many different optical imperfections simultaneously. Apart from providing a new simulation method, the proposed theoretical framework with a "perturbation" perspective has the potential to provide physical insights into the deterioration of the performance that results from the various types of optical errors. 
Although we have only dealt with one set of optical systems in this work, the proposed theory can also deal with successive optical elements. The distorted CSD function after one optical element is treated as the ideal CSD function for the analysis of the next optical element. Notice that the coordinates $\mathbf{r}_{1}$ and $\mathbf{r}_{2}$ in the 2D FFT term in the convolution operation are already decoupled. The coherent mode decomposition can used for successive optical elements as well.

\section{ACKNOWLEDGEMENT}

We would like to thank Dr Lucia Alianelli and Dr David Laundy (DLS) for their fruitful discussions and suggestions. Dr Yiming Yang and Dr Guangcai Chang (BSRF) are also thanked for their discussions on the practical application of the proposed theory to the beamline design.

\section{REFERENCES}

[1] “Diamond-II Conceptual Design Report.” <https://www.diamond.ac.uk/Home/About/Vision/Diamond-II.html> .

[2] D. Chenevier, and A. Joly, "ESRF: Inside the Extremely Brilliant Source Upgrade," Synchrotron Radiation News 31, 32-35 (2018).

[3] Y. Jiao, G. Xu, X.-H. Cui, Z. Duan, Y.-Y. Guo, P. He, D.-H. Ji, J.-Y. Li, X.-Y. Li, C. Meng, Y.-M. Peng, S.-K. Tian, J.-Q. Wang, N. Wang, Y.-Y. Wei, H.-S. Xu, F. Yan, C.-H. Yu, Y.-L. Zhao, and Q. Qin, "The HEPS project," Journal of Synchrotron Radiation 25, 1611-1618 (2018).

[4] S. C. Leemann, M. Sjöström, and Å. Andersson, "First optics and beam dynamics studies on the MAX IV 3 $\mathrm{GeV}$ storage ring," Nuclear Instruments and Methods in Physics Research Section A: Accelerators, Spectrometers, Detectors and Associated Equipment 883, 33-47 (2018).

[5] Xianbo Shi, Ruben Reininger, Ross Harder, Dean Haeffner, "X-ray optics simulation and beamline design for the APS upgrade," Proc. SPIE 10388, Advances in Computational Methods for X-Ray Optics IV, 103880C (2017).

[6] O. Chubar, Y. S. Chu, K. Kaznatcheev, and H. Yan, "Application of partially coherent wavefront propagation calculations for design of coherence-preserving synchrotron radiation beamlines," Nuclear Instruments and Methods in Physics Research Section A: Accelerators, Spectrometers, Detectors and Associated Equipment 649, 118-122 (2011).

[7] J. Bahrdt, "Wavefront tracking within the stationary phase approximation," Physical Review Special Topics Accelerators and Beams 10, 060701 (2007).

[8] Luca Rebuffi, Manuel Sanchez del Rio, "OASYS (OrAnge SYnchrotron Suite): an open-source graphical environment for x-ray virtual experiments," Proc. SPIE 10388, Advances in Computational Methods for X-Ray Optics IV, 103880S (2017).

[9] X. Shi, R. Reininger, M. Sanchez del Rio, and L. Assoufid, "A hybrid method for X-ray optics simulation: combining geometric ray-tracing and wavefront propagation," Journal of Synchrotron Radiation 21, 669-678 (2014).

[10] Konstantin Klementiev, Roman Chernikov, "Powerful scriptable ray tracing package xrt," Proc. SPIE 9209, Advances in Computational Methods for X-Ray Optics III, 92090A (2014).

[11] M. Sanchez del Rio, R. Celestre, M. Glass, G. Pirro, J. R. Herrera, R. Barrett, J. C. Da Silva, P. Cloetens, X. Shi, and L. Rebuffi, "A hierarchical approach for modeling X-ray beamlines: application to a coherent beamline," Journal of synchrotron radiation 26 (2019).

[12]M. Glass, and M. S. del Rio, "Coherent modes of X-ray beams emitted by undulators in new storage rings," EPL (Europhysics Letters) 119, 34004 (2017)

[13] L. Mandel, and E. Wolf, [Optical coherence and quantum optics] Cambridge university press (1995).

[14] M. Born, and E. Wolf, [Principles of optics: electromagnetic theory of propagation, interference and diffraction of light] Elsevier (2013).

[15] J. W. Goodman, [Introduction to Fourier Optics] W. H. Freeman (2005)

[16] C. G. Schroer, and G. Falkenberg, "Hard X-ray nanofocusing at low-emittance synchrotron radiation sources," Journal of synchrotron radiation 21, 996-1005 (2014).

[17]I. A. Vartanyants, and A. Singer, "Coherence properties of hard x-ray synchrotron sources and x-ray freeelectron lasers," New Journal of Physics 12, 035004 (2010). 